\title{
Comment on a Paper: “Ben-Naim's 'Pitfalls': Don Quixote's Windmill”, by Y. Fang, Open Journal of Biophysics, 2013, 3, 13-21
}

\author{
Arieh Ben-Naim \\ Department of Physical Chemistry, The Hebrew University of Jerusalem, Jerusalem, Israel \\ Email: arieh@fh.huji.ac.il
}

Received February 28, 2013; revised April 5, 2013; accepted May 7, 2013

Copyright (C) 2013 Arieh Ben-Naim. This is an open access article distributed under the Creative Commons Attribution License, which permits unrestricted use, distribution, and reproduction in any medium, provided the original work is properly cited.

\begin{abstract}
This article is a response to Fang's article which criticizes statements never made by Ben-Naim and never appeared in the literature.
\end{abstract}

Keywords: Protein Folding; Leviathan Paradox

\section{Introduction}

The article by Fang [1] deals with purported claims attributed to Ben-Naim's articles [2-4]. Unfortunately, the whole article is based on statements, presented as if they were quotations from Ben-Naim's articles, when in fact, were never ever made by Ben-Naim.

To start with, the title of the article is ambiguous. Does the author mean that Ben-Naim is Don Quixote, or that the "pitfalls" discussed in the articles are the "Windmill"?

In the following pages, I shall present only a few examples. Interested readers can find many more examples in this article [1].

\section{In the Abstract the Author States}

"Ben-Naim, in three articles dismissed and 'answered' the Levinthal paradox..."

Indeed, I dismissed the Levinthal paradox in my articles [2,5], but I never "answered" the "Levinthal paradox". I was careful enough to make it clear that I was answering the Levinthal question [4], and not the paradox [2]. This is an important distinction overlooked and misunderstood by the author.

\section{In the Abstract the Author States}

"He claims (Ben-Naim) no existence of Gibbs energy formula $G(X)$."

This is not true. In all my writings I discussed the function $G(X)$, and I never said that such a formula does not exist! For more details, the reader is referred to my recent monograph [6].

\section{In the Abstract and in the Article the Author Claims}

"His minimum distribution $P_{e q}$ is wrong."

I never said that. What I was talking about is the distribution $P_{e q}$ that minimizes the Gibbs energy functional (see below). I have no idea what the "minimum distribution $P_{e q}$ is, which the author claims to be "mine."

\section{In Section 1 of the Article, the Author Writes}

"Here Ben-Naim implies that the conformation of a protein should not be the variable of the Gibbs energy."

Again, this is not true. The so-called Gibbs energy landscape is a function of the conformation of the protein. I never claimed, nor implied that the "conformation of a protein should not be the variable of the Gibbs energy”.

\section{Another Quotation from Section 1 of the Article [1]}

“Even knowing what is Ben-Naim's minimum distribution, we still do not know what the three dimensional shape of the native structure.”

Again, I never talked about "Ben-Naim's minimum distribution”. I have no idea what the "Ben-Naim's minimum distribution” is. As I noted above what I talk about 
is the distribution $P_{e q}$ which minimizes the Gibbs energy functional, under a given environment $(T, P, N)$. If one knew this distribution, then one could tell which conformations are more probable than the others under the given environment.

\section{In Section 2, the Author Writes}

"All previous attempts of deriving the Gibbs energy formula, including Ben-Naim's, missed the goal of identifying the three dimensional structure of a native protein.”

This quotation is doubly misleading. In a previous quotation above, the author claims that Ben-Naim "claims no existence of Gibbs energy formula”. If I claim that "Gibbs energy formula does not exist" (which I never did, see above), how then could I even attempt (among others), to derive the Gibbs free energy formula? In this quotation the author contradicts his previous statements. The truth is that I never denied the existence of the Gibbs energy formula, nor did I ever attempt to derive the Gibbs free energy formula. Therefore, all of the criticisms are addressed to claims that were never made by me in any of my writings.

\section{Finally, in His Conclusion the Author Writes}

“Ben-Naim's minimization at $P_{e q}(R)$ is analyzed and dismissed because it predicts that at equilibrium every possible conformation $R$ will have the same probability.”

This is again a criticism of a claim I never made. The parameter $R$ in $P_{e q}(R)$ is the location of the center of a simple spherical solute in a solution. This is clearly stated in my article. The function $P_{e q}(R)=\frac{1}{V}$ is the equilibrium density function for the locations of the solute particle. This is an exact result. It has nothing to do with proteins! Yet the author criticism is "Ben-Naim claims that $P_{e q}(R)=\frac{1}{V}$ for any conformation $R$ ”, a claim attributed to me but which I never made.

There are many more misquotations and false statements in this article. I believe the examples provided above suffice to demonstrate the character of this article. It is also clear that the author of Reference [1] did not understand any of my articles

I should end with a note on the author's claim of achievements.

Finally, the author claims that he demonstrates how "to apply quantum statistics to derive the Gibbs energy formula $G(X)$, and the folding force". Both of these are not delivered. All he did was to rewrite the Gibbs energy function $G(X)$ in terms of the entropy function $S(X)$, the volume function $V(X)$ and the energy function $U(X)$ but not an explicit Gibbs energy function $G(X)$. His achievement is summarized in Section 7.4 as:

"Thus, by (27) we obtain the Gibbs free energy $G(X) \ldots$

$$
\begin{aligned}
G(X) & =P V(X)+U(X)-T S(X),, \\
& =\sum_{i=1} \mu_{i} N_{i}(X)+\mu_{c} N_{c}(X)
\end{aligned}
$$

Which is a well known identity.

\section{Conclusion}

This article does not provide anything new, and the criticisms it raises are addressed to statements that I never made.

\section{REFERENCES}

[1] Y. Fang, “'Ben-Naim's "Pitfall’: Don Quixote's Windmill,” Open Journal of Biophysics, Vol. 3, No. 1, 2013, pp. 13-21. http://dx.doi.org/10.4236/ojbiphy.2013.31002

[2] C. Levinthal, "How to Fold Graciously,” Mössbauer Spectroscopy in Biological Systems Proceedings, Vol. 67, No. 41, 1969, pp. 22-26.

[3] A. Ben-Naim, "Levinthal's Paradox Revisited and Dismissed,” Open Journal of Biophysics, Vol. 2, No. 2, 2012, pp. 22-32. http://dx.doi.org/10.4236/ojbiphy.2012.22004

[4] A. Ben-Naim, "Pitfalls in Anfinsen's Thermodynamic Hypothesis,” Chemical Physics Letters, Vol. 511, No. 1-3, 2011, pp. 126-128.

http://dx.doi.org/10.1016/j.cplett.2011.05.049

[5] A. Ben-Naim, "Levinthal's Question Revisited, and Answered," Journal of Biomolecular Structure and Dynamics, Vol. 30, No. 1, 2012, pp. 113-124. http://dx.doi.org/10.1080/07391102.2012.674286

[6] A. Ben-Naim, "The Protein Folding Problem and Its Solutions,” World Scientific, Singapore, 2013. http://dx.doi.org/10.1142/8627 